\title{
Multiple solutions for Kirchhoff elliptic equations in Orlicz-Sobolev spaces
}

\section{Shujun $\mathrm{Wu}^{*}$}

\section{"Correspondence:}

wushujun@upc.edu.cn

College of Science, China University

of Petroleum, Qingdao, 266580,

China

\begin{abstract}
We investigate the existence of solutions of the Kirchhoff elliptic equations with nonlinearity in $R^{N}$. Using the ideas developed in Orlicz spaces and the technique of variation principle, we prove that there are at least three solutions in Orlicz-Sobolev spaces.
\end{abstract}

MSC: 35J60; 35J70; 46B20; 58E05

Keywords: Kirchhoff elliptic equation; Orlicz-Sobolev spaces; Orlicz function; multiple solutions

\section{Introduction}

The equation

$$
\rho \frac{\partial^{2} u}{\partial t^{2}}-\left(\frac{P_{0}}{h}+\frac{E}{2 L} \int_{0}^{L}\left|\frac{\partial u}{\partial x}\right|^{2} d x\right) \frac{\partial^{2} u}{\partial x^{2}}=0
$$

presented by Kirchhoff [1] in 1883, is an extension of the classical d'Alembert's wave equation by considering the changes in the length of the string during vibrations. In (1.1), $L$ is the length of string, $h$ is the area of the cross section, $E$ is the Young modulus of the material, $\rho$ is the mass density, and $P_{0}$ is the initial tension. The stationary form of type (1.1) is taken in the form of

$$
-k\left(\int_{\Omega}|\nabla u|^{2} d x\right) \Delta u=f(x, u), \quad \text { in } \Omega
$$

which may be used for modeling several physical and biological systems, where $u$ describes a process which depends on the average of itself, for example, the population density [2]. If $k\left(\int_{\Omega}|\nabla u|^{2} d x\right)$ is replaced by $k\left(\int_{\Omega}|u|^{2} d x\right)$, then the equation

$$
-k\left(\int_{\Omega}|u|^{2} d x\right) \Delta u=f(x, u), \quad \text { in } \Omega
$$

arises in numerous physical models such as systems of particles in thermodynamical equilibrium via gravitational potential, thermal runaway in ohmic heating and shear bands in metal deformed under high strain rates [3].

(c) The Author(s) 2017. This article is distributed under the terms of the Creative Commons Attribution 4.0 International License (http://creativecommons.org/licenses/by/4.0/), which permits unrestricted use, distribution, and reproduction in any medium, provided you give appropriate credit to the original author(s) and the source, provide a link to the Creative Commons license, and indicate if changes were made. 
Ricceri [4] discussed Kirchhoff equations in the form of

$$
\begin{cases}-k\left(\int_{\Omega}|\nabla u|^{2} d x\right) \Delta u=\lambda f(x, u)+\mu g(x, u), & \text { in } \Omega, \\ u=0, & \text { on } \partial \Omega .\end{cases}
$$

The existence of solutions was established by the critical point theorem due to Ricceri [5]. Chen [6] investigated $p$-Kirchhoff equations in the whole space $R^{N}$ :

$$
\begin{cases}M\left(\int_{R^{N}}|\nabla u|^{p}+V(x)|u|^{p} d x\right)\left(-\triangle_{p} u+V(x)|u|^{p-2} u\right)=f(x, u)+g(x), & \text { in } R^{N}, \\ u \rightarrow 0, & \text { as }|x| \rightarrow \infty\end{cases}
$$

where $M(t)=t^{k}, k>0$ and $V(x)$ is a continuous function satisfying the condition that there exists $b_{0}>0$ such that $V(x) \geq b_{0}$ for $x \in R^{N}, V(x) \rightarrow \infty$ as $|x| \rightarrow \infty$. The solutions were obtained by the mountain pass theorem, Ekeland's variational principle and Krasnoselskii's genus theory. Also Chen [7] considered (1.5) with $M(t)=a+b t^{k}(a>0, b>0, k>0)$, $f(x, u)=\lambda h_{1}(x)|u|^{q-2} u+h_{2}(x)|u|^{r-2} u+h_{3}(x)$ and $g(x)=0$. Two nontrivial solutions were established by the mountain pass theorem and Ekeland's variational principle.

There often arise the equations involving nonhomogeneous operators and nonlinearities, for instance, in the fields of electrorheological fluids (sometimes referred to as 'smart fluids'), nonlinear elasticity and plasticity. The natural setting for this approach is OrliczSobolev spaces.

In this article, we investigate Kirchhoff elliptic equations with nonlinearity in $R^{N}$ in the Orlicz-Sobolev setting:

$$
\begin{cases}-k\left(\widetilde{\rho}_{M}(u)\right)(\operatorname{div}(a(|\nabla u|) \nabla u)-a(|u|) u)=\lambda f(x, u)+\mu g(x, u), & \text { in } R^{N}, \\ u \rightarrow 0, & \text { as }|x| \rightarrow \infty,\end{cases}
$$

where $k:[0, \infty) \rightarrow[0, \infty)$ is nondecreasing and continuous, $a(t) t$ is strictly increasing and continuous on $[0, \infty)$, such that $\lim _{t \rightarrow 0^{+}} a(t) t=0, \lim _{t \rightarrow \infty} a(t) t=\infty, \lambda, \mu$ are nonnegative real numbers, $f$ and $g$ are Carathéodory functions, and $\widetilde{\rho}_{M}$ is defined in Section 2.

Using the ideas and techniques developed in the research of Orlicz spaces, we prove the existence of at least three solutions of (1.6) but get rid of the restriction that $M(\sqrt{t})$ is convex on $[0, \infty)$ in $[8-13]$.

To our knowledge, this is the first contribution to investigate (1.6) in the whole space $R^{N}$ in the Orlicz-Sobolev setting. For multivalued problems, please see [14]. For problems in variable exponent, please see [15-17]. For hyperbolic Kirchhoff systems, please see [18, 19].

\section{Preliminaries}

$M$ is called an Orlicz function provided that

$$
M(s)=\int_{0}^{|s|} p(t) d t, \quad \forall s \in R,
$$

where $p$ is nondecreasing, right-continuous with $p(0)=0, p(t)>0(\forall t>0)$ and $\lim _{t \rightarrow \infty} p(t)=\infty$. Its complementary function $\widetilde{M}$ is defined as

$$
\widetilde{M}(t)=\int_{0}^{|t|} \widetilde{p}(s) d s, \quad \forall t \in R
$$

where $\widetilde{p}(s)=\sup \{t \geq 0: p(t) \leq s\}$. Then $\widetilde{M}$ is also an Orlicz function (see [20]). 
Young's inequality holds true

$$
s t \leq M(s)+\tilde{M}(t), \quad \forall s, t \geq 0,
$$

and equation holds if and only if $t=p(s)$ or $s=\widetilde{p}(t)$.

$M$ is said to satisfy $\Delta_{2}$-condition (in short, $M \in \Delta_{2}$ ) provided that there exists a positive number $C>0$ such that

$$
M(2 t) \leq C M(t), \quad \forall t \geq 0 .
$$

We use $M \in \nabla_{2}$ to stand for $\widetilde{M} \in \Delta_{2}$.

Denote

$$
d_{M}:=\inf _{t>0} \frac{t p(t)}{M(t)}, \quad D_{M}:=\sup _{t>0} \frac{t p(t)}{M(t)}, \quad d_{M}^{*}:=\frac{N d_{M}}{N-d_{M}}, \quad D_{M}^{*}:=\frac{N D_{M}}{N-D_{M}} .
$$

For a measurable function $u: R^{N} \rightarrow R$, denoted as $u \in \widetilde{L}$, we define

$$
\rho_{M}(u)=\int_{R^{N}} M(u(x)) d x, \quad \tilde{\rho}_{M}(u)=\rho_{M}(u)+\rho_{M}(|\nabla u|) .
$$

The Orlicz space $L_{M}\left(R^{N}\right)$ (in short $L_{M}$ ) is defined by

$$
L_{M}\left(R^{N}\right)=\left\{u \in \widetilde{L}: \exists \lambda>0, \rho_{M}(\lambda u)<\infty\right\}
$$

endowed with the Luxemburg norm

$$
\|u\|_{(M), R^{N}}=\|u\|_{(M)}=\inf \left\{\lambda>0: \rho_{M}\left(\frac{u}{\lambda}\right) \leq 1\right\},
$$

or with the Orlicz norm

$$
\|u\|_{M, R^{N}}=\|u\|_{M}=\sup \left\{\int_{R^{N}} u(x) v(x) d x: \int_{R^{N}} \tilde{M}(v(x)) d x \leq 1\right\} .
$$

Then $\left(L_{M},\|\cdot\|_{(M)}\right)$ and $\left(L_{M},\|\cdot\|_{M}\right)$ form Banach spaces (see [20, 21]).

For $u \in L_{M}, v \in L_{\widetilde{M}}$, the Hölder inequality holds (see [20])

$$
\int_{R^{N}} u(x) v(x) d x \leq\|u\|_{M}\|v\|_{(\widetilde{M})} .
$$

The Sobolev conjugate $M_{*}$ of $M$ is defined by

$$
M_{*}^{-1}(t)=\int_{0}^{t} \frac{M^{-1}(s)}{s^{\frac{N+1}{N}}} d s, \quad t \geq 0
$$

provided $\int_{0}^{1} \frac{M^{-1}(t)}{t^{\frac{N+1}{N}}} d t<\infty, \int_{1}^{\infty} \frac{M^{-1}(t)}{t^{\frac{N+1}{N}}} d t=\infty$ (see [22]).

An Orlicz-Sobolev space $W^{1, M}\left(R^{N}\right)$ (in short $W^{1, M}$ ) is defined by

$$
W^{1, M}\left(R^{N}\right)=\left\{u \in L_{M}\left(R^{N}\right): D^{\alpha} u \in L_{M}\left(R^{N}\right),|\alpha| \leq 1\right\}
$$


endowed with

$$
\|u\|=\|u\|_{W^{1, M}}=\inf \left\{\lambda>0: \widetilde{\rho}_{M}\left(\frac{u}{\lambda}\right) \leq 1\right\} .
$$

Then $\left(W^{1} L_{M},\|\cdot\|_{W^{1} L_{M}}\right.$ ) forms a Banach space (refer to [22]).

If $M \in \Delta_{2} \cap \nabla_{2}$, then $L_{M}$ and $W^{1} L_{M}$ are separable and reflexive Banach spaces (refer to [22]).

Let $W_{0}^{1, M}\left(R^{N}\right)$ (in short $W_{0}^{1, M}$ ) be the closure of $C_{c}^{\infty}\left(R^{N}\right)$, that is, the set of all functions which are differentiable for any order and with compact support in $W^{1, M}\left(R^{N}\right), W_{0}^{1, M}\left(R^{N}\right)=$ $W^{1, M}\left(R^{N}\right)$ (refer to [22]).

\section{Main results}

In this section, we firstly give the main result of this paper. Secondly, we prove some lemmas for the main result. Finally, we establish the existence of at least three solutions in Orlicz-Sobolev spaces applying the variation principle.

For convenience, we set several conditions.

$k(0): \quad k(t) \geq C t^{\gamma}, \forall t \in(0, \delta)$, for some $C>0, \delta>0, \gamma \in\left(0, \frac{d_{M}^{*}}{D_{M}}-1\right)$.

$k(\infty): k(t) \geq C t^{\gamma}, \forall t \geq \delta$, for some $C>0, \delta>0, \gamma>0$.

$H_{1}: \quad|f(x, t)| \leq \alpha(x) p(|t|)+\beta(x), \forall(x, t) \in R^{N} \times R$, for some nonnegative functions $\alpha(x) \in$ $L^{\infty}\left(R^{N}\right) \cap L^{\frac{D_{M}^{*}}{D_{M}^{*} d_{M}}}\left(R^{N}\right), \beta(x) \in L_{\widetilde{M}}\left(R^{N}\right) \cap L^{\infty}\left(R^{N}\right)$.

$H_{2}: \quad \lim \sup _{t \rightarrow 0} \frac{F(x, t)}{M_{*}(t)}<\infty$, uniformly $x \in R^{N}$.

$H_{3}: \quad \int_{R^{N}}\left(\int_{0}^{u_{0}(x)} f(x, s) d s\right) d x>0$ for some $u_{0} \in W_{0}^{1, M}$.

In the following, we always assume that $k:[0, \infty) \rightarrow[0, \infty)$ is nondecreasing and $k(t)>0$ $(\forall t>0)$.

For $u \in W_{0}^{1, M}$, we define

$$
\begin{aligned}
K(u) & =\int_{0}^{\widetilde{\rho}_{M}(u)} k(s) d s, \\
\mathcal{F}(u) & =\int_{R^{N}}\left(\int_{0}^{u(x)} f(x, s) d s\right) d x=\int_{R^{N}} F(x, u(x)) d x, \\
\mathcal{G}(u) & =\int_{R^{N}}\left(\int_{0}^{u(x)} g(x, s) d s\right) d x .
\end{aligned}
$$

Definition 3.1 $u \in W_{0}^{1, M}$ is called a weak solution of problem (1.6) if, for all $v \in W_{0}^{1, M}$,

$$
k\left(\widetilde{\rho}_{M}(u)\right) \int_{\Omega}[a(|\nabla u|) \nabla u \cdot \nabla v+a(|u|) u v] d x=\lambda \int_{\Omega} f(x, u) v d x+\mu \int_{\Omega} g(x, u) v d x .
$$

The main result of this paper is as follows.

Theorem 3.1 Let $1<d_{M} \leq D_{M}<N$ and $D_{M}<d_{M}^{*}$. Assume that $k$ satisfies $k(0)$ and $k(\infty)$, $f$ satisfies $H_{1}, H_{2}, H_{3}$ and $g$ satisfies $H_{1}$. Then, for all $[a, b] \subset(0, \infty)$, there exists $\sigma>0$ such that for each $\lambda \in[a, b]$ there exists $\delta>0$ such that for each $\mu \in[0, \delta]$, the problem

$$
\begin{cases}-k\left(\tilde{\rho}_{M}(u)\right)(\operatorname{div}(a(|\nabla u|) \nabla u)-a(|u|) u)=\lambda f(x, u)+\mu g(x, u), & \text { in } R^{N}, \\ u \rightarrow 0, & \text { as }|x| \rightarrow \infty\end{cases}
$$


has at least three solutions whose norms are less than $\sigma$ in an Orlicz-Sobolev space $W_{0}^{1, M}$ for a strictly convex and smooth Orlicz function $M(t)=\int_{0}^{|t|} a(\tau) \tau d \tau$.

\section{Proof of the main results}

In the proof of Lemmas 4.1 and 4.2, we get rid of the restriction that $M(\sqrt{t})$ is convex on $[0, \infty)$ as in [8, Theorem 3.1], [9, Lemma 3.2], [10, Lemma 5], [12, Lemma 2.4], in which by this condition a Clarkson-type inequality was established in order to show that a sequence strongly converges. Here, we get the convergent sequence by using the ideas and techniques developed in Orlicz spaces research without the help of Clarkson-type inequality.

Lemma 4.1 Given that $M$ is strictly convex and $M \in \Delta_{2}$. For $\left\{u_{n}\right\}$, a sequence weakly converging to $u$ in $W_{0}^{1} L_{M}$, and $\lim _{n \rightarrow \infty} K\left(u_{n}\right)=K(u)$, we have that $\left\{u_{n}\right\}$ strongly converges to $u$ in $W_{0}^{1} L_{M}$.

Proof Since $u_{n} \rightarrow u$ weakly and $M \in \Delta_{2}$, by [9, Lemma 2.3], we assume $\lim _{n \rightarrow \infty} \widetilde{\rho}_{M}\left(u_{n}\right)=$ $d \geq 0$, if necessary passing to a subsequence. By $\lim _{n \rightarrow \infty} K\left(u_{n}\right)=K(u)$ and $\int_{0}^{t} k(s) d s$ is strictly increasing with respect to $t>0$, we obtain

$$
\lim _{n \rightarrow \infty} \widetilde{\rho}_{M}\left(u_{n}\right)=\widetilde{\rho}_{M}(u)
$$

and $\widetilde{\rho}_{M}\left(\frac{u_{n}+u}{2}\right) \leq \frac{\widetilde{\rho}_{M}\left(u_{n}\right)+\widetilde{\rho}_{M}(u)}{2} \rightarrow \widetilde{\rho}_{M}(u)$. Therefore, $\lim \sup _{n \rightarrow \infty} \widetilde{\rho}_{M}\left(\frac{u_{n}+u}{2}\right) \leq \widetilde{\rho}_{M}(u)$.

By the convexity of $M$ and [23, Theorem 25.20], $K$ is sequentially weakly lower semicontinuous. Since $u_{n} \rightarrow u$ weakly, it follows that $\frac{u_{n}+u}{2} \rightarrow u$ weakly and $\liminf _{n \rightarrow \infty} K\left(\frac{u_{n}+u}{2}\right) \geq$ $K(u)$. Consequently, $\liminf _{n \rightarrow \infty} \widetilde{\rho}_{M}\left(\frac{u_{n}+u}{2}\right) \geq \widetilde{\rho}_{M}(u)$, and thus

$$
\lim _{n \rightarrow \infty} \tilde{\rho}_{M}\left(\frac{u_{n}+u}{2}\right)=\widetilde{\rho}_{M}(u)
$$

If $u=\theta$ (that is, $\theta(x)=0$, $\mu$-a.e. $x \in R^{N}$ ), thanks to $M \in \Delta_{2}$, (4.1) implies $\lim _{n \rightarrow \infty} \widetilde{\rho}_{M}\left(u_{n}\right)=$ $0=\lim _{n \rightarrow \infty}\left\|u_{n}\right\|$.

If $u \neq \theta$, then $\widetilde{\rho}_{M}(u)>0$ and $\left\|u_{n}\right\|>0$.

Claim $\left\{u_{n}\right\}$ converges to $u$ in measure.

Otherwise, we suppose that for some $\varepsilon_{0}>0, \sigma_{0}>0$, there exists a subsequence $\left\{u_{n_{j}}\right\}$ such that $\mu\left\{x \in R^{N}:\left|u_{n_{j}}(x)-u(x)\right| \geq \sigma_{0}\right\} \geq \varepsilon_{0}>0$. Set $c=M^{-1}\left(\frac{6 \widetilde{\rho}_{M}(u)}{\varepsilon_{0}}\right)>0$, we get that

$$
\begin{aligned}
\widetilde{\rho}_{M}(u) & =\int_{R^{N}}[M(u(x))+M(|\nabla u(x)|)] d x \geq \int_{R^{N}} M(u(x)) d x \\
& \geq \int_{|u(x)|>c} M(u(x)) d x \geq M(c) \mu\left\{x \in R^{N}:|u(x)|>c\right\} \\
& =\frac{6 \widetilde{\rho}_{M}(u)}{\varepsilon_{0}} \mu\left\{x \in R^{N}:|u(x)|>c\right\} .
\end{aligned}
$$

The above inequality yields $\mu\left\{x \in R^{N}:|u(x)|>c\right\} \leq \frac{\varepsilon_{0}}{6}<\frac{\varepsilon_{0}}{3}$. 
From (4.1), there exists $j_{0}$, for all $j \geq j_{0}$,

$$
\begin{aligned}
2 \widetilde{\rho}_{M}(u) & \geq \widetilde{\rho}_{M}\left(u_{n_{j}}\right) \geq \int_{R^{N}} M\left(u_{n_{j}}(x)\right) d x \geq \int_{\left|u_{n_{j}}(x)\right|>c} M\left(u_{n_{j}}(x)\right) d x \\
& \geq M(c) \mu\left\{x \in R^{N}:\left|u_{n_{j}}(x)\right|>c\right\}=\frac{6 \widetilde{\rho}_{M}(u)}{\varepsilon_{0}} \mu\left\{x \in R^{N}:\left|u_{n_{j}}(x)\right|>c\right\} .
\end{aligned}
$$

Consequently, for all $j \geq j_{0}$, we obtain that $\mu\left\{x \in R^{N}:\left|u_{n_{j}}(x)\right|>c\right\} \leq \frac{\varepsilon_{0}}{3}$.

Since

$$
\begin{aligned}
\{x \in & \left.R^{N}:\left|u_{n_{j}}(x)-u(x)\right| \geq \sigma_{0}\right\} \\
\subset & \left\{x \in R^{N}:|u(x)|>c\right\} \\
& \cup\left\{x \in R^{N}:\left|u_{n_{j}}(x)\right|>c\right\} \\
& \cup\left\{x \in R^{N}:\left|u_{n_{j}}(x)-u(x)\right| \geq \sigma_{0},|u(x)| \leq c,\left|u_{n_{j}}(x)\right| \leq c\right\},
\end{aligned}
$$

one has

$$
\begin{aligned}
\mu E & :=\mu\left\{x \in R^{N}:\left|u_{n_{j}}(x)-u(x)\right| \geq \sigma_{0},|u(x)| \leq c,\left|u_{n_{j}}(x)\right| \leq c\right\} \\
& \geq \varepsilon_{0}-\frac{\varepsilon_{0}}{3}-\frac{\varepsilon_{0}}{3}=\frac{\varepsilon_{0}}{3} .
\end{aligned}
$$

Since $M(t)$ is strictly convex, from [24, Proposition 1.4], there exists $\delta>0$ such that

$$
M\left(\frac{t^{\prime}+t^{\prime \prime}}{2}\right) \leq(1-\delta) \frac{M\left(t^{\prime}\right)+M\left(t^{\prime \prime}\right)}{2}
$$

for all $\left|t^{\prime}\right| \leq c,\left|t^{\prime \prime}\right| \leq c$ and $\left|t^{\prime}-t^{\prime \prime}\right| \geq \sigma_{0}$. By (4.3) and (4.4),

$$
\begin{aligned}
\widetilde{\rho}_{M}\left(\frac{u_{n_{j}}+u}{2}\right) \leq & \int_{E}(1-\delta) \frac{M\left(u_{n_{j}}(x)\right)+M(u(x))}{2} d x+\int_{R^{N} \backslash E} \frac{M\left(u_{n_{j}}(x)\right)+M(u(x))}{2} d x \\
& +\int_{R^{N}} \frac{M\left(\left|\nabla u_{n_{j}}(x)\right|\right)+M(|\nabla u(x)|)}{2} d x \\
\leq & \frac{\widetilde{\rho}_{M}\left(u_{n_{j}}\right)+\widetilde{\rho}_{M}(u)}{2}-\delta M\left(\frac{\sigma_{0}}{2}\right) \frac{\varepsilon_{0}}{3} .
\end{aligned}
$$

Letting $j \rightarrow \infty$, by (4.1) and (4.2) we obtain $\widetilde{\rho}_{M}(u) \leq \frac{\widetilde{\rho}_{M}(u)+\widetilde{\rho}_{M}(u)}{2}-\delta M\left(\frac{\sigma_{0}}{2}\right) \frac{\varepsilon_{0}}{3}<\widetilde{\rho}_{M}(u)$, which is a contradiction.

Replacing $u_{n}, u$ by $\nabla u_{n}, \nabla u$, respectively, we deduce that $\left\{\nabla u_{n}\right\}$ converges to $\nabla u$ in measure.

By the Riesz theorem, there exists a subsequence, still denoted by $\left\{u_{n_{j}}\right\}$, such that $u_{n_{j}}(x) \rightarrow u(x), \nabla u_{n_{j}}(x) \rightarrow \nabla u(x)$ a.e. in $R^{N}$, so $\left|\nabla u_{n_{j}}(x)\right| \rightarrow|\nabla u(x)|$ a.e. in $R^{N}$. Applying 
the Fatou lemma and (4.1), we get that

$$
\begin{aligned}
\tilde{\rho}_{M}(u)= & \int_{R^{N}}[M(u(x))+M(|\nabla u(x)|)] d x \\
= & \int_{R^{N}} \lim _{j \rightarrow \infty}\left[\frac{M\left(u_{n_{j}}(x)\right)+M(u(x))}{2}-M\left(\frac{u_{n_{j}}(x)-u(x)}{2}\right)\right. \\
& \left.+\frac{M\left(\left|\nabla u_{n_{j}}(x)\right|\right)+M(|\nabla u(x)|)}{2}-M\left(\frac{\left|\nabla u_{n_{j}}(x)-\nabla u(x)\right|}{2}\right)\right] d x \\
\leq & \liminf _{j \rightarrow \infty} \int_{R^{N}}\left[\frac{M\left(u_{n_{j}}(x)\right)+M(u(x))}{2}-M\left(\frac{u_{n_{j}}(x)-u(x)}{2}\right)\right. \\
& \left.+\frac{M\left(\left|\nabla u_{n_{j}}(x)\right|\right)+M(|\nabla u(x)|)}{2}-M\left(\frac{\left|\nabla u_{n_{j}}(x)-\nabla u(x)\right|}{2}\right)\right] d x \\
= & \widetilde{\rho}_{M}(u)-\limsup _{j \rightarrow \infty} \int_{R^{N}}\left[M\left(\frac{u_{n_{j}}(x)-u(x)}{2}\right)+M\left(\frac{\left|\nabla u_{n_{j}}(x)-\nabla u(x)\right|}{2}\right)\right] d x \\
= & \widetilde{\rho}_{M}(u)-\limsup _{j \rightarrow \infty} \widetilde{\rho}_{M}\left(\frac{u_{n_{j}}-u}{2}\right) .
\end{aligned}
$$

Hence, $\limsup _{j \rightarrow \infty} \widetilde{\rho}_{M}\left(\frac{u_{n_{j}}-u}{2}\right) \leq 0$. Combining $\liminf _{j \rightarrow \infty} \widetilde{\rho}_{M}\left(\frac{u_{n_{j}}-u}{2}\right) \geq 0$, we see that $\lim _{j \rightarrow \infty} \widetilde{\rho}_{M}\left(\frac{u_{n_{j}}-u}{2}\right)=0$. From $M \in \Delta_{2}$, we obtain $\lim _{j \rightarrow \infty}\left\|u_{n_{j}}-u\right\|=0$. Arguing by contradiction, we deduce that $\lim _{n \rightarrow \infty}\left\|u_{n}-u\right\|=0$.

Lemma 4.2 Given that $k$ is continuous, $M$ is a strictly convex smooth Orlicz function and $M \in \Delta_{2} \cap \nabla_{2}$. Then $\left(K^{\prime}\right)^{-1}:\left(W_{0}^{1, M}\right)^{*} \rightarrow W_{0}^{1, M}$ is continuous.

Proof For $u, v \in W_{0}^{1, M}$, by [25, Lemma 3.4], we obtain

$$
\left\langle K^{\prime}(u), v\right\rangle=k\left(\widetilde{\rho}_{M}(u)\right) \int_{R^{N}}\left[p(|\nabla u|) \frac{\nabla u \cdot \nabla v}{|\nabla u|}+p(|u|) \frac{u v}{|u|}\right] d x
$$

$K^{\prime}: W_{0}^{1, M} \rightarrow\left(W_{0}^{1, M}\right)^{*}$ and $K \in C^{1}\left(W_{0}^{1, M}, R\right)$.

Next, we show that $K^{\prime}$ is strictly monotone.

For $u_{1}, u_{2} \in W_{0}^{1, M}$ with $u_{1} \neq u_{2}$, it follows that $\mu A:=\mu\left\{x \in R^{N}: u_{1}(x) \neq u_{2}(x)\right\}>0$. Since $M$ is strictly convex, we get that

$$
\begin{aligned}
& \int_{A} M\left(\frac{u_{1}(x)+u_{2}(x)}{2}\right) d x<\int_{A} \frac{M\left(u_{1}(x)\right)+M\left(u_{2}(x)\right)}{2} d x \\
& \int_{R^{N} \backslash A}\left[\frac{M\left(u_{1}(x)\right)+M\left(u_{2}(x)\right)}{2}-M\left(\frac{u_{1}(x)+u_{2}(x)}{2}\right)\right] d x \geq 0,
\end{aligned}
$$

and

$$
\int_{R^{N}}\left[\frac{M\left(\left|\nabla u_{1}(x)\right|\right)+M\left(\left|\nabla u_{2}(x)\right|\right)}{2}-M\left(\frac{\left|\nabla u_{1}(x)+\nabla u_{2}(x)\right|}{2}\right)\right] d x \geq 0
$$

The above inequalities yield $\widetilde{\rho}_{M}\left(\frac{u_{1}+u_{2}}{2}\right)<\frac{\widetilde{\rho}_{M}\left(u_{1}\right)+\widetilde{\rho}_{M}\left(u_{2}\right)}{2}$. By [23, Theorem 25.8 ], we get that $K^{\prime}$ is strictly monotone. 
By (4.5), we deduce that $K^{\prime}$ is coercive and hemi-continuous. In view of [23, Theorem 26.A], $\left(K^{\prime}\right)^{-1}:\left(W_{0}^{1, M}\right)^{*} \rightarrow W_{0}^{1, M}$ is strictly monotone, bounded and demi-continuous.

Finally, we show that $\left(K^{\prime}\right)^{-1}$ is continuous on $\left(W_{0}^{1, M}\right)^{*}$.

For $\left\{\tau_{n}\right\} \subset\left(W_{0}^{1, M}\right)^{*}, \tau \in\left(W_{0}^{1, M}\right)^{*}$, if $\left\|\tau_{n}-\tau\right\| \rightarrow 0$, we will prove that $\left\|u_{n}-u\right\| \rightarrow 0$, where $u_{n}=\left(K^{\prime}\right)^{-1}\left(\tau_{n}\right), u=\left(K^{\prime}\right)^{-1}(\tau)$.

Since $\left(K^{\prime}\right)^{-1}$ is demi-continuous, it follows that $\left\langle K^{\prime}\left(u_{n}\right)-K^{\prime}(u), u_{n}-u\right\rangle=\left\langle\tau_{n}-\tau\right.$, $\left.u_{n}-u\right\rangle \rightarrow 0$. By $K^{\prime}(u) \in\left(W_{0}^{1} L_{M}\right)^{*}$, it follows that

$$
\begin{aligned}
& \left\langle K^{\prime}(u), u_{n}-u\right\rangle \rightarrow 0, \\
& \left\langle K^{\prime}\left(u_{n}\right), u_{n}-u\right\rangle=\left\langle K^{\prime}\left(u_{n}\right)-K^{\prime}(u), u_{n}-u\right\rangle+\left\langle K^{\prime}(u), u_{n}-u\right\rangle \rightarrow 0 .
\end{aligned}
$$

Claim $\widetilde{\rho}_{M}\left(u_{n}\right) \rightarrow \widetilde{\rho}_{M}(u)$, as $n \rightarrow \infty$.

Since $M$ is an Orlicz function, by (4.5) we obtain

$$
\begin{aligned}
& \left\langle K^{\prime}(u), u_{n}-u\right\rangle \\
& \quad=k\left(\widetilde{\rho}_{M}(u)\right) \int_{R^{N}}\left[p(|\nabla u|) \frac{\nabla u \cdot \nabla\left(u_{n}-u\right)}{|\nabla u|}+p(|u|) \frac{u\left(u_{n}-u\right)}{|u|}\right] d x \\
& \quad \leq k\left(\widetilde{\rho}_{M}(u)\right) \int_{R^{N}}\left[p(|\nabla u|)\left(\left|\nabla u_{n}\right|-|\nabla u|\right)+p(|u|)\left(\left|u_{n}\right|-|u|\right)\right] d x \\
& \quad \leq k\left(\widetilde{\rho}_{M}(u)\right)\left(\widetilde{\rho}_{M}\left(u_{n}\right)-\widetilde{\rho}_{M}(u)\right), \\
& \left\langle K^{\prime}\left(u_{n}\right), u_{n}-u\right\rangle \\
& \quad=k\left(\widetilde{\rho}_{M}\left(u_{n}\right)\right) \int_{R^{N}}\left[p\left(\left|\nabla u_{n}\right|\right) \frac{\nabla u_{n} \cdot \nabla\left(u_{n}-u\right)}{\left|\nabla u_{n}\right|}+p\left(\left|u_{n}\right|\right) \frac{u_{n}\left(u_{n}-u\right)}{\left|u_{n}\right|}\right] d x \\
& \quad \geq k\left(\widetilde{\rho}_{M}\left(u_{n}\right)\right) \int_{R^{N}}\left[p\left(\left|\nabla u_{n}\right|\right)\left(\left|\nabla u_{n}\right|-|\nabla u|\right)+p\left(\left|u_{n}\right|\right)\left(\left|u_{n}\right|-|u|\right)\right] d x \\
& \quad \geq k\left(\widetilde{\rho}_{M}\left(u_{n}\right)\right)\left(\widetilde{\rho}_{M}\left(u_{n}\right)-\widetilde{\rho}_{M}(u)\right) .
\end{aligned}
$$

If $u=\theta$, then $\widetilde{\rho}_{M}(u)=0$. (4.7) and (4.9) yield $0 \leq k\left(\widetilde{\rho}_{M}\left(u_{n}\right)\right) \widetilde{\rho}_{M}\left(u_{n}\right) \leq\left\langle K^{\prime}\left(u_{n}\right), u_{n}\right\rangle \rightarrow 0$. Because of the positivity of $k(t)$, we obtain $\widetilde{\rho}_{M}\left(u_{n}\right) \rightarrow 0=\widetilde{\rho}_{M}(u)$.

If $u \neq \theta$, then $\tilde{\rho}_{M}(u)>0$. From (4.6) and (4.8), we deduce that $\widetilde{\rho}_{M}\left(u_{n}\right)-\widetilde{\rho}_{M}(u) \geq$ $\frac{\left\langle K^{\prime}(u), u_{n}-u\right\rangle}{k\left(\widetilde{\rho}_{M}(u)\right)}$. Letting $n \rightarrow \infty$, one has $\liminf _{n \rightarrow \infty}\left(\widetilde{\rho}_{M}\left(u_{n}\right)-\widetilde{\rho}_{M}(u)\right) \geq 0$. Combining (4.7) and (4.9), we deduce that there exists $n_{0}$, for all $n \geq n_{0}$,

$$
\widetilde{\rho}_{M}\left(u_{n}\right)-\widetilde{\rho}_{M}(u) \leq \frac{\left\langle K^{\prime}\left(u_{n}\right), u_{n}-u\right\rangle}{k\left(\widetilde{\rho}_{M}\left(u_{n}\right)\right)} \leq \frac{\left|\left\langle K^{\prime}\left(u_{n}\right), u_{n}-u\right\rangle\right|}{k\left(\frac{\widetilde{\rho}_{M}(u)}{2}\right)} \rightarrow 0 .
$$

Thus, we have $\lim \sup _{n \rightarrow \infty}\left(\widetilde{\rho}_{M}\left(u_{n}\right)-\widetilde{\rho}_{M}(u)\right) \leq 0$. Consequently, $\lim _{n \rightarrow \infty} \widetilde{\rho}_{M}\left(u_{n}\right)=\widetilde{\rho}_{M}(u)$. Moreover, $\lim _{n \rightarrow \infty} K\left(u_{n}\right)=K(u)$. By Lemma 4.1, we obtain $\left\|u_{n}-u\right\| \rightarrow 0$.

Lemma 4.3 Given that $M \in \Delta_{2} \cap \nabla_{2}$ with $D_{M}<N$, $f$ satisfies $H_{1}$ and $\alpha(x) \in L^{\frac{D_{M}^{*}}{D_{M}^{*} d_{M}}}\left(R^{N}\right)$. Then $D_{M}<d_{M}^{*}$ implies that $\mathcal{F}^{\prime}$ is compact. 
Proof Using the same arguments as those in [25, Lemma 3.4], we get that

$$
\left\langle\mathcal{F}^{\prime}(u), v\right\rangle=\int_{R^{N}} f(x, u(x)) v(x) d x
$$

$\mathcal{F}^{\prime}: W_{0}^{1, M} \rightarrow\left(W_{0}^{1, M}\right)^{*}, \mathcal{F} \in C^{1}\left(W_{0}^{1, M}, R\right)$.

For $\left\{u_{n}\right\}$ a bounded sequence in $W_{0}^{1} L_{M}$, by the Alaoglu-Banach theorem, there exists a subsequence $\left\{u_{n_{j}}\right\}$ such that $u_{n_{j}} \rightarrow u$ weakly. We will show that $\left\{\mathcal{F}^{\prime}\left(u_{n}\right)\right\}$ admits a subsequence strongly converging to $\mathcal{F}^{\prime}(u)$.

By $M \in \Delta_{2}$ and the Hölder inequality, for any measurable subset $\Omega \subset R^{N}$, it holds uniformly for all $v \in W_{0}^{1, M}$ with $\|v\| \leq 1$,

$$
\begin{aligned}
& \int_{\Omega}[\alpha(x) p(|u(x)|)+2 \beta(x)] v(x) d x \\
& \quad \leq\|\alpha p(|u|)+2 \beta\|_{\widetilde{M}, \Omega}\|v\|_{(M), \Omega} \leq\|\alpha p(|u|)+2 \beta\|_{\widetilde{M}, \Omega^{\prime}} \\
& \int_{\Omega} \alpha(x) p\left(\left|u_{n_{j}}(x)\right|\right) v(x) d x \leq\left\|\alpha p\left(\left|u_{n_{j}}\right|\right)\right\|_{\widetilde{M}, \Omega} .
\end{aligned}
$$

By (4.10), for $\varepsilon>0$, there exists $r^{*}>0, \Omega^{*}:=\left\{x \in R^{N}:|x|>r^{*}\right\}$ such that

$$
\int_{\Omega^{*}}[\alpha(x) p(|u(x)|)+2 \beta(x)] v(x) d x<\varepsilon
$$

Due to $M, \widetilde{M} \in \Delta_{2}$, by [24, Theorem 1.13], it follows that $p, \widetilde{p} \in \Delta_{2}$. We deduce that

$$
\begin{aligned}
& \widetilde{M}\left(\alpha(x) p\left(\left|u_{n_{j}}(x)\right|\right)\right) \\
& \quad \leq \alpha(x) p\left(\left|u_{n_{j}}(x)\right|\right) \widetilde{p}\left(\alpha(x) p\left(\left|u_{n_{j}}(x)\right|\right)\right) \leq \alpha(x) p\left(\left|u_{n_{j}}(x)\right|\right) \widetilde{p}\left(\|\alpha\|_{\infty} p\left(\left|u_{n_{j}}(x)\right|\right)\right) \\
& \quad \leq \alpha(x) p\left(\left|u_{n_{j}}(x)\right|\right) \widetilde{p}\left(2\|\alpha\|_{\infty}\left(p\left(\left|u_{n_{j}}(x)\right|\right)-\varepsilon(x)\right)\right) \\
& \quad \leq c_{1}^{\prime} \alpha(x) p\left(\left|u_{n_{j}}(x)\right|\right) \widetilde{p}\left(p\left(\left|u_{n_{j}}(x)\right|\right)-\varepsilon(x)\right) \\
& \quad \leq c_{1}^{\prime} \alpha(x) p\left(\left|u_{n_{j}}(x)\right|\right)\left|u_{n_{j}}(x)\right| \leq c_{1}^{\prime} \alpha(x) M\left(2\left|u_{n_{j}}(x)\right|\right) \leq c_{1} \alpha(x) M\left(\left|u_{n_{j}}(x)\right|\right),
\end{aligned}
$$

where $\varepsilon(x)>0$ is taken small enough for $u_{n_{j}}(x) \neq 0, \varepsilon(x)=0$ for $u_{n_{j}}(x)=0$.

By (4.13), $D_{M}<d_{M}^{*}$ and [26, Lemma 2.1, Lemma 2.2],

$$
\begin{aligned}
& \int_{\left|u_{n_{j}}(x)\right| \leq 1} \tilde{M}\left(\alpha(x) p\left(\left|u_{n_{j}}(x)\right|\right)\right) d x \\
& \leq c_{1} \int_{\left|u_{n_{j}}(x)\right| \leq 1} \alpha(x) M\left(\left|u_{n_{j}}(x)\right|\right) d x \leq c_{1} \int_{\left|u_{n_{j}}(x)\right| \leq 1} \alpha(x) M(1)\left|u_{n_{j}}(x)\right|^{d_{M}} d x \\
& \leq c_{1} M(1)\left(\int_{\left|u_{n_{j}}(x)\right| \leq 1} \alpha(x)^{\frac{D_{M}^{*}}{D_{M}^{*}-d_{M}}} d x\right)^{\frac{D_{M}^{*}-d_{M}}{D_{M}^{*}}}\left(\int_{\left|u_{n_{j}}(x)\right| \leq 1}\left|u_{n_{j}}(x)\right|^{d_{M} \cdot \frac{D_{M}^{*}}{d_{M}}} d x\right)^{\frac{d_{M}}{D_{M}^{*}}}
\end{aligned}
$$

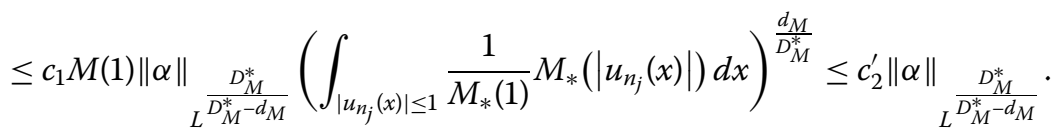


Using the same arguments, we obtain

$$
\int_{\left|u_{n_{j}}(x)\right|>1} \tilde{M}\left(\alpha(x) p\left(\left|u_{n_{j}}(x)\right|\right)\right) d x \leq c_{2}^{\prime \prime}\|\alpha\| \frac{d_{M}^{*}}{L^{d_{M}^{*}-D_{M}}} .
$$

Combining the above inequalities, taking $r \geq r^{*}, \Omega_{r}=\left\{x \in R^{N}:|x|>r\right\}$, we deduce that $\int_{\Omega_{r}} \widetilde{M}\left(\alpha(x) p\left(\left|u_{n_{j}}(x)\right|\right)\right) d x<\varepsilon, j=1,2, \ldots$

By [26, Lemma 2.5] and (4.11),

$$
\int_{\Omega_{r}} \alpha(x) p\left(\left|u_{n_{j}}(x)\right|\right) v(x) d x \leq\left\|\alpha p\left(\left|u_{n_{j}}\right|\right)\right\|_{\widetilde{M}, \Omega_{r}} \leq 2 \varepsilon^{\frac{d_{M^{-1}}}{d_{M}}}, \quad j=1,2, \ldots
$$

By $H_{1},(4.12)$ and (4.14), we deduce that it holds uniformly for all $v \in W_{0}^{1, M}$ with $\|v\| \leq 1$

$$
\begin{aligned}
& \int_{\Omega_{r}}\left[f\left(x, u_{n_{j}}(x)\right)-f(x, u(x))\right] v(x) d x \\
& \quad \leq \int_{\Omega_{r}}\left[\alpha(x) p\left(\left|u_{n_{j}}(x)\right|\right)+\alpha(x) p(|u(x)|)+2 \beta(x)\right]|v(x)| d x \\
& \quad<2 \varepsilon^{\frac{d_{M-1}}{d_{M}}}+\varepsilon, \quad j=1,2, \ldots
\end{aligned}
$$

For $B_{r}=R^{N} \backslash \Omega_{r}=\left\{x \in R^{N}:|x| \leq r\right\}$, combining $u_{n_{j}} \rightarrow u$ weakly, we get $\left\|u_{n_{j}}-u\right\|_{(M), B_{r}} \rightarrow 0$. By applying the Lebesgue dominated convergence theorem, we deduce $\int_{B_{r}} \widetilde{M}\left(f\left(x, u_{n_{j}}(x)\right)-f(x, u(x))\right) d x \rightarrow 0$. By $\widetilde{M} \in \Delta_{2}$, there exists $j_{0}$, for all $j \geq j_{0}$,

$$
\left\|f\left(x, u_{n_{j}}\right)-f(x, u)\right\|_{\widetilde{M}, B_{r}}<\varepsilon
$$

In view of (4.15) and (4.16), we complete the proof.

Lemma 4.4 Given that $d_{M}<N$ and $D_{M}<d_{M}^{*}$, $k$ satisfies $k(0)$ and $k(\infty)$, $f$ satisfies $H_{1}, H_{2}$. Then $\lim \sup _{\|u\| \rightarrow \infty} \frac{\mathcal{F}(u)}{K(u)} \leq 0, \lim \sup _{\|u\| \rightarrow 0} \frac{\mathcal{F}(u)}{K(u)} \leq 0$.

Proof Given $\delta$ in Condition $k(\infty)$. For $\|u\|>1+\delta$, so $\widetilde{\rho}_{M}(u)>1+\delta$.

By $H_{1}$, the Hölder inequality and $k(\infty)$,

$$
\begin{aligned}
\frac{\mathcal{F}(u)}{K(u)} & \leq \frac{\int_{R^{N}}[\alpha(x) M(|u(x)|)+\beta(x)|u(x)|] d x}{\int_{\delta}^{\widetilde{\rho}_{M}(u)} k(s) d s} \\
& \leq \frac{\|\alpha\|_{\infty} \widetilde{\rho}_{M}(u)+\|\beta\|_{\tilde{M}}\|u\|}{\frac{C}{\gamma+1}\left(\left(\widetilde{\rho}_{M}(u)\right)^{\gamma+1}-(\delta)^{\gamma+1}\right)} \leq \frac{\left(\|\alpha\|_{\infty}+\|\beta\|_{\widetilde{M}}\right) \widetilde{\rho}_{M}(u)}{\frac{C}{\gamma+1}\left(\left(\widetilde{\rho}_{M}(u)\right)^{\gamma+1}-(\delta)^{\gamma+1}\right)},
\end{aligned}
$$

which implies lim $\sup _{\|u\| \rightarrow \infty} \frac{\mathcal{F}(u)}{K(u)} \leq 0$.

By $H_{2}$, for some positive constants $\delta_{0} \in(0,1), c_{1}>0$, one has $F(x, t) \leq c_{1} M_{*}(t)$ for all $|t|<\delta_{0}$. Due to $W_{0}^{1} L_{M}\left(R^{N}\right) \hookrightarrow L_{M_{*}}\left(R^{N}\right)$, there exists $C_{*}>0$ such that $\|u\|_{\left(M_{*}\right)} \leq C_{*}\|u\|$ for all $u \in W_{0}^{1} L_{M}\left(R^{N}\right)$. For $\|u\|<\min \left\{1, \frac{1}{C_{*}}, \delta^{\prime}\right\}$ (where $\delta^{\prime}$ is of in $k(0)$ ), by [26, Lemma 2.2], we obtain $\rho_{M_{*}}(u) \leq\|u\|_{\left(M_{*}\right)}^{d_{M}^{*}} \leq C_{*}^{d_{M}^{*}}\|u\|^{d_{M}^{*}}$.

By $D_{M}<d_{M}^{*}$ and Condition $k(0)$, we get that

$$
\frac{\int_{|u(x)|<\delta_{0}} F(x, u(x)) d x}{K(u)} \leq \frac{c_{1} \rho_{M_{*}}(u)}{\frac{C}{\gamma+1}\left(\widetilde{\rho}_{M}(u)\right)^{\gamma+1}} \leq \frac{c_{1} C_{*}^{d_{M}^{*}}(\gamma+1)}{C}\|u\|^{d_{M}^{*}-D_{M}(\gamma+1)} \rightarrow 0 .
$$


Since $M$ is an Orlicz function, combining [26, Lemma 2.2], there exists $c_{2}>0$ such that

$$
\int_{|u(x)| \geq \delta_{0}} F(x, u(x)) d x \leq c_{2} \int_{|u(x)| \geq \delta_{0}}|u(x)|^{d_{M}^{*}} d x .
$$

By $1>\int_{R^{N}} M(u(x)) d x \geq M\left(\delta_{0}\right) \mu\left\{x \in R^{N}:|u(x)| \geq \delta_{0}\right\}$, we obtain $\mu\left\{x \in R^{N}:|u(x)| \geq \delta_{0}\right\} \leq$ $\frac{1}{M\left(\delta_{0}\right)}<\infty$. From [22, Theorem 8.12] and $t^{d_{M}^{*}} \leq \frac{1}{M_{*}(1)} M_{*}(t), \forall t \geq 1$, there exists $c_{3}>0$ such that, for all $v \in L^{d_{M}^{*}}\left(\left\{|u(x)| \geq \delta_{0}\right\}\right)$,

$$
\|v\|_{L^{M_{M}^{*}}\left(\left\{|u(x)| \geq \delta_{0}\right\}\right)} \leq c_{3}\|v\|_{\left(M_{*}\right),\left\{|u(x)| \geq \delta_{0}\right\}} \leq c_{3} C_{*}\|v\| .
$$

Combining $k(0)$ and (4.18)-(4.19), we have

$$
\begin{aligned}
\frac{\int_{|u(x)| \geq \delta_{0}} F(x, u(x)) d x}{K(u)} & \leq \frac{c_{2} \int_{|u(x)| \geq \delta_{0}}|u(x)|^{d_{M}^{*}} d x}{\frac{C}{\gamma+1}\left(\widetilde{\rho}_{M}(u)\right)^{\gamma+1}} \\
& \leq \frac{c_{2}\left(c_{3} C_{*}\right)_{M}^{d_{M}^{*}}(\gamma+1)}{C}\|u\|^{d_{M}^{*}-D_{M}(\gamma+1)} \rightarrow 0 .
\end{aligned}
$$

By (4.17) and (4.20), it follows that $\lim \sup _{\|u\| \rightarrow 0} \frac{\mathcal{F}(u)}{K(u)} \leq 0$.

Lemma 4.5 ([5]) Let $X$ be a separable and reflexive real Banach space; let $\Phi: X \rightarrow R$ be a coercive, sequentially weakly lower semi-continuous $C^{1}$ functional, belonging to $\mathcal{W}_{X}$ (if $\left\{u_{n}\right\}$ is a sequence in $X$ converging weakly to $u$ and $\liminf _{n \rightarrow \infty} \Phi\left(u_{n}\right) \leq \Phi(u)$, then $\left\{u_{n}\right\}$ has a subsequence converging strongly to $u$ ), bounded on each bounded subset of $X$ and whose derivative admits a continuous inverse on $X^{*}$; let $J: X \rightarrow R$ be a $C^{1}$ functional with compact derivative. Assume that $\Phi$ has a local minimum $x_{0}$ with $\Phi\left(x_{0}\right)=J\left(x_{0}\right)=0$. Finally, setting

$$
\begin{aligned}
& \alpha=\max \left\{0, \limsup _{\|x\| \rightarrow+\infty} \frac{J(x)}{\Phi(x)}, \limsup _{x \rightarrow x_{0}} \frac{J(x)}{\Phi(x)}\right\}, \\
& \beta=\sup _{x \in \Phi^{-1}((0,+\infty))} \frac{J(x)}{\Phi(x)},
\end{aligned}
$$

assume that $\alpha<\beta$.

Then, for each compact interval $[a, b] \subset\left(\frac{1}{\beta}, \frac{1}{\alpha}\right)$ (with the conventions $\frac{1}{0}=+\infty, \frac{1}{+\infty}=0$ ), there exists $r>0$ with the following property: for each $\lambda \in[a, b]$ and every $C^{1}$ functional $\Psi$ with compact derivative, there exists $\delta>0$ such that, for each $\mu \in[0, \delta]$, the equation

$$
\Phi^{\prime}(x)=\lambda J^{\prime}(x)+\mu \Psi^{\prime}(x)
$$

has at least three solutions whose norms are less than $r$.

Now, we give the proof of the main result.

Proof Let $p(t)=a(t) t, \forall t>0$, and $M(s)=\int_{0}^{|s|} p(t) d t, \forall s \in R$. Then $M$ is a strictly convex smooth Orlicz function.

Applying Lemmas 4.1 and 4.2, we deduce that $K$ is sequentially weakly lower semicontinuous belonging to the class $\mathcal{W}_{W_{0}^{1, M}}$ and $\left(K^{\prime}\right)^{-1}$ is continuous. In view of Lemma 4.3, 
we deduce that $\mathcal{F}, \mathcal{G} \in C^{1}\left(W_{0}^{1, M}, R\right)$ with compact derivatives. From the definitions of $K$ and $\mathcal{F}$, we see that $K(\theta)=\mathcal{F}(\theta)=0$ and $K(u)>0$ for all $u \neq \theta$. By Lemma 4.4 and $H_{3}$, it follows that $\beta>\alpha$.

Summarily, the critical points theorem in Lemma 4.5 guarantees that

$$
K^{\prime}(u)=\lambda \mathcal{F}^{\prime}(u)+\mu \mathcal{G}^{\prime}(u)
$$

has at least three solutions, which implies that Problem (1.6) has at least three solutions.

\section{Corollaries and examples}

By Theorem 3.1, we get the following.

Corollary 5.1 Let $1<d_{M} \leq D_{M}<N$ and $D_{M}<d_{M}^{*}$. Assume that $k$ satisfies $k(0)$ and $k(\infty)$, f satisfies $H_{1}, H_{2}^{\prime}, H_{3}$

$$
H_{2}^{\prime}: \limsup _{t \rightarrow 0} \frac{f(x, t)}{t|t|^{D_{M}^{*}-2}}<\infty, \quad \text { uniformly } x \in R^{N},
$$

and $g$ satisfies $H_{1}$. Then, for all $[a, b] \subset(0, \infty)$, there exists $\sigma>0$ such that for each $\lambda \in[a, b]$ there exists $\delta>0$ such that for each $\mu \in[0, \delta]$, the problem

$$
\begin{cases}-k\left(\widetilde{\rho}_{M}(u)\right)(\operatorname{div}(a(|\nabla u|) \nabla u)-a(|u|) u)=\lambda f(x, u)+\mu g(x, u), & \text { in } R^{N}, \\ u \rightarrow 0, & \text { as }|x| \rightarrow \infty\end{cases}
$$

has at least three solutions whose norms are less than $\sigma$ in an Orlicz-Sobolev space $W_{0}^{1, M}$ for a strictly convex and smooth Orlicz function $M(t)=\int_{0}^{|t|} a(\tau) \tau d \tau$.

The following example shows that Theorem 3.1 is a substantial improvement, where the function $M$ listed below satisfies the conditions of Theorem 3.1 but it does not satisfy those of [8, Theorem 3.1], [9, Lemma 3.2], [10, Lemma 5], [12, Lemma 2.4].

Example 5.2 For $p \in(1,2)$,

$$
M(t):=|t|^{p}
$$

Then $M$ satisfies all the assumptions in Theorem 3.1. However, the function $M(\sqrt{t})=t^{\frac{p}{2}}$ is not convex w.r.t. $t \in[0, \infty)$ due to $\frac{1}{2}<\frac{p}{2}<1$.

\section{Conclusion}

We prove the existence of three solutions for the Kirchhoff elliptic problem (1.6) using the ideas developed in Orlicz spaces and the technique of variation principle. We point out that the convexity of the Orlicz function $M(\sqrt{t})$, which is required in [8-10,12], is unnecessary during the proof of strong convergence. We also notice that the result in Theorem 3.1 extends the result for bounded domains. 
Funding

Not applicable.

\section{Competing interests}

The author declares that they have no competing interests.

\section{Authors' contributions}

All authors conceived of the study, participated in its design and coordination, drafted the manuscript, participated in the sequence alignment, and read and approved the final manuscript.

\section{Publisher's Note}

Springer Nature remains neutral with regard to jurisdictional claims in published maps and institutional affiliations.

Received: 25 May 2017 Accepted: 26 August 2017 Published online: 08 September 2017

\section{References}

1. Kirchhoff, G: Mechanik. Teubner, Leipzig (1883)

2. Alves, CO, Corrêa, FJSA, Ma, TF: Positive solutions for a quasilinear elliptic equation of Kirchhoff type. Comput. Math. Appl. 49, 85-93 (2005)

3. Aouaoui, S: Multiplicity result for some nonlocal anisotropic equation via nonsmooth critical point theory approach. Appl. Math. Comput. 218, 532-541 (2011)

4. Ricceri, B: On an elliptic Kirchhoff-type problem depending on two parameters. J. Glob. Optim. 46(4), $543-549$ (2010)

5. Ricceri, B: A further refinement of a three critical points theorem. Nonlinear Anal. 71, 4151-4157 (2009)

6. Chen, CS, Song, HX, Xiu, ZH: Multiple solutions for $p$-Kirchhoff equations in $R^{N}$. Nonlinear Anal. 86, 146-156 (2013)

7. Chen, CS, Huang, JC, Liu, LH: Multiple solutions to the nonhomogeneous $p$-Kirchhoff elliptic equation with concave-convex nonlinearities. Appl. Math. Comput. 26, 754-759 (2013)

8. Cammaroto, F, Vilasi, L: Multiple solutions for a nonhomogeneous Dirichlet problem in Orlicz-Sobolev spaces. Appl. Math. Comput. 218, 11518-11527 (2012)

9. Kristály, A, Mihăilescu, M, Rădulescu, V: Two nontrivial solutions for a nonhomogeneous Neumann problem: an Orlicz-Sobolev setting. Proc. R. Soc. Edinb. 139A, 367-379 (2009)

10. Mihăilescu, M, Repovš, D: Multiple solutions for a nonlinear and nonhomogeneous problem in Orlicz-Sobolev spaces Appl. Math. Comput. 217, 6624-6632 (2011)

11. Chung, NT: Multiple solutions for a nonlocal problem in Orlicz-Sobolev spaces. Ric. Mat. 63, 169-182 (2014)

12. Chung, NT: Three solutions for a class of nonlocal problems in Orlicz-Sobolev spaces. J. Korean Math. Soc. 50(6), 1257-1269 (2013)

13. Aouaoui, S: On some eigenvalue problem involving a non-homogeneous differential operator. Complex Var. Elliptic Equ. 58(10), 1421-1429 (2013)

14. Figueiredo, GJM, Santos, JA: On a $\Phi$-Kirchhoff multivalued problem with critical growth in an Orlicz-Sobolev space. Asymptot. Anal. 89(1-2), 151-172 (2014)

15. Radulescu, V, Repovs, D: Partial Differential Equation with Variable Exponents: Variable Method and Qualitative Analysis. CRC Press, Boca Raton (2015)

16. Radulescu, V: Nonlinear elliptic equations with variable exponent: old and new. Nonlinear Anal. 121, 336-369 (2015)

17. Repovs, D: Stationary waves of Schrodinger-type equations with variable exponent. Anal. Appl. 13(6), 645-661 (2015)

18. Autuori, G, Pucci, P: Kirchhoff systems with nonlinear source and boundary damping terms. Commun. Pure Appl. Anal. 9(5), 1161-1188 (2010)

19. Pucci, P, Xiang, M, Zhang, B: Existence and multiplicity of entire solutions for fractional p-Kirchhoff equations. Adv. Nonlinear Anal. 5(1), 27-55 (2016)

20. Krasnoselski, M, Rutickii, Y: Convex Functions and Orlicz Space. Noordhoff, Groningen (1961)

21. Dominguez, T, Hudzik, H, López, G, Mastyło, M, Sims, B: Complete characterizations of Kadec-Klee properties in Orlicz spaces. Houst. J. Math. 29(4), 1027-1044 (2003)

22. Adams, RA, Fournier, JJF: Sobolev Spaces, 2nd edn. Academic Press, New York (2003)

23. Zeidler, E: Nonlinear Functional Analysis and Its Applications. Springer, New York (1990)

24. Chen, ST: Geometry of Orlicz Spaces. Polish Sci., Warszawa (1996)

25. García-Huidobro, M, Le, VK, Manásevich, R, Schmitt, K: On principal eigenvalues for quasilinear elliptic differential operators: an Orlicz-Sobolev space setting. Nonlinear Differ. Equ. Appl. 6, 207-225 (1999)

26. Fukagai, N, Ito, M, Narukawa, K: Positive solutions of quasilinear elliptic equations with critical Orlicz-Sobolev nonlinearity on $R^{N}$. Funkc. Ekvacioj 49, 235-267 (2006) 